\title{
Collective excitations of trapped imbalanced fermion gases
}

\author{
Achilleas Lazarides and Bert Van Schaeybroeck \\ Instituut voor Theoretische Fysica, Katholieke Universiteit Leuven, Celestijnenlaan 200 D, B-3001 Leuven, Belgium
}

(Received 5 September 2007; published 2 April 2008)

\begin{abstract}
We present a theoretical study of the collective excitations of a trapped imbalanced fermion gas at unitarity, when the system consists of a superfluid core and a normal outer shell. We formulate the relevant boundary conditions and treat the normal shell both hydrodynamically and collisionlessly. For an isotropic trap, we calculate the mode frequencies as a function of trap polarization. Out-of-phase modes with frequencies below the trapping frequency are obtained for the case of a hydrodynamic normal shell. For the collisionless case, we calculate the monopole mode frequencies and find that all but the lowest mode may be damped.
\end{abstract}

DOI: 10.1103/PhysRevA.77.041602

PACS number(s): 03.75.Ss, 05.30.Fk, 03.75.Kk, 47.37.+q

The recent pioneering experiments on polarized fermion systems in the Bose-Einstein-condensation (BEC)-BCS crossover make a broad range of exciting new phenomena accessible and offer the opportunity to study longstanding issues in the field of strongly interacting many-body systems $[1,2]$. By loading a trap with unequal numbers of particles of two spin states, a phase-separated system consisting of an unpolarized superfluid (SF) core and a surrounding polarized normal $(\mathrm{N})$ phase is obtained. Experimentally probing the collective excitation frequencies in ultracold gases is, by now, a standard technique, and has proved indispensable to understanding such systems. An experimental study of collective modes in imbalanced fermionic superfluid systems in the near future seems, therefore, a realistic prospect.

The N-SF interface plays a major role in the static properties of the polarized system: taking into account the interface tension has proved essential to explaining the experiments performed in highly anisotropic traps [3,4]. Moreover, its presence affects the thermal equilibration process at low temperatures [5] and has been speculated to cause large fluctuations in the polarization at the interface [6].

In this Rapid Communication, we study the effects of polarization on the frequencies of the collective excitations. To do this, it is necessary first to establish the boundary conditions encoding the important physics at the interface. We then study two distinct cases: in one, the normal part is assumed to behave hydrodynamically, and in the other, collisionlessly, so that it is described by a Boltzmann-Vlasov equation. We present results for isotropic traps only; results for a highly elongated trap will be presented separately, along with more detailed calculations [7].

For a fully hydrodynamic trapped system, we find two general classes of excitations: A first class consists of modes which for zero polarization reduce to the well-known singlecomponent-system excitations; for finite polarization, these evolve to modes with the same general character of motion but slightly shifted frequencies. In what follows, we refer to them as in-phase (IP) modes, for reasons that will become clear. Additionally, excitations unique to the two-component system constitute a second class. Particularly interesting is that, at low polarization, these modes have frequencies below the trapping frequency. Each of these low-frequency excitations is in one-to-one correspondence with the nodeless $(n=0, \ell)$ modes of the single-component system, to which their motion is related as follows: the outer boundary of the
$\mathrm{N}$ and SF components oscillate in the same way as the corresponding one-component $n=0$ mode, except that they are out of phase with each other. This is illustrated in Fig. 1 for the quadrupole mode. For brevity, we refer to these types of excitations as the out-of-phase (OOP) modes.

In the collisionless case, we study the monopole $(\ell=0)$ modes. Simultaneously solving the hydrodynamic and Boltzmann-Vlasov equations for the SF and N phases, respectively, and imposing the appropriate boundary conditions, we find that the lowest, $n=1$, monopole mode remains at $2 \omega_{0}$ for all polarizations (here $\omega_{0}$ is the trapping frequency), in agreement with an exact result obtained by Castin [8]. On varying the polarization, the $n=2$ monopole mode frequency crosses over from the value appropriate for a fully superfluid system at zero polarization and a fully collisionless system at complete polarization. For this (and higher) monopole modes, and for some intermediate polarizations, damping occurs, the cause of which is purely geometric.

We shall first describe the equilibrium state of the system, and then separately present our calculations and results for the hydrodynamic and collisionless cases.

Consider a spin mixture of polarization $\left(N_{\uparrow}-N_{\downarrow}\right) /\left(N_{\uparrow}\right.$ $+N_{\downarrow}$ ) with $N_{\uparrow}$ and $N_{\downarrow}$ the spin up and down particle numbers, trapped by a harmonic potential $V(\mathbf{r})$. A nonzero imbalance generally results in the appearance of at least two phases: the unpolarized SF and the partially or completely polarized $\mathrm{N}$ phase which consists, in general, of a mixture of $\downarrow$ and $\uparrow$ particles.

When the $\mathrm{N}$ and SF phases are separated by a first-order phase transition, an interface is formed between them with the SF in the trap center. Due to the universal nature of the strongly interacting regime, the equilibrium pressure $\bar{P}$ and density $\bar{\rho}$ in the SF are known [9] to be related by $\bar{P}_{S F}$ $=\hbar^{2} \xi\left(3 \pi^{2}\right)^{2 / 3} \bar{\rho}_{S F}^{5 / 3} / 5 m$, with the universal constant $\xi \approx 0.41$ and $m$ the particle mass; in the $\mathrm{N}$ phase, on the other hand,

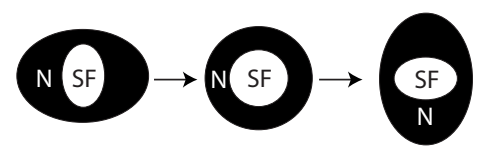

FIG. 1. Time evolution of the quadrupole $(\ell=2)$ out-of-phase mode of an imbalanced fermion system in the hydrodynamicnormal-gas case. The outer boundaries of the normal $(\mathrm{N})$ and the superfluid (SF) phase perform the same motion, but $\pi$ out of phase. 
one has $\bar{P}_{i}=\hbar^{2}\left(6 \pi^{2}\right)^{2 / 3} \bar{\rho}_{i}^{5 / 3} / 5 m$ for $i=\uparrow, \downarrow$ (the overline denotes equilibrium values). Moreover, within the local density approximation all three of $\uparrow, \downarrow$, and SF may be described by an effective equilibrium chemical potential $\bar{\mu}_{j}(\mathbf{r})=\mu_{j}^{0}-V(\mathbf{r})$, where $\mu_{j}^{0}$ is the chemical potential at the center of the trap and $j=\uparrow, \downarrow$, or SF [10].

Next we include the interface into our equilibrium framework. At zero temperature, the SF is theoretically predicted to be unpolarized; experimentally, the trap core is observed to be unpolarized. This implies a negligible population of single-particle excitations in the trap center at experimental temperatures. Denoting the equilibrium position of the interface by $\bar{\zeta}$, chemical equilibrium is then ensured by imposing [11]

$$
2 \bar{\mu}_{S F} \mid \bar{\zeta}=\left(\bar{\mu}_{\uparrow}+\bar{\mu}_{l}\right) \bar{\zeta} .
$$

Furthermore, for mechanical stability, the Laplace condition must be satisfied:

$$
\left(\bar{P}_{S F}-\bar{P}_{\uparrow}-\bar{P}_{\downarrow}\right)_{\bar{\zeta}}=\left.\bar{\sigma}\left(\frac{1}{R_{1}}+\frac{1}{R_{2}}\right)\right|_{\bar{\zeta}} .
$$

Here, $R_{1}$ and $R_{2}$ are the radii of curvature of the interface, the position of which is denoted by $\zeta$, and $\sigma$ is the interface tension [3,4]; following Haque and Stoof [4], we take $\sigma$ $=0.6 m \mu_{S F}^{2} / \hbar^{2}[12]$. Note that $\mu_{S F}$ is the total chemical potential, including its fluctuation during an oscillation, which reduces to $\bar{\mu}_{S F}$ at equilibrium. Equations (1) and (2), together with the expressions for the densities and pressures, allow us to fix the position of the interface for given particle number and polarization in the global equilibrium state. To do this, all that is necessary is to solve Eqs. (1) and (2) for the radii while imposing the total particle number to be $7 \times 10^{6}$. We then find that, for all polarizations, the superfluid core is surrounded by a fully polarized normal shell of the majority species. This is similar to what is observed in the Rice experiments $[2,4]$.

Dynamical phenomena, such as the collective excitations studied here, involve situations in which the local velocities are nonzero. For such cases, involving departures from the global equilibrium state, we must extend Eqs. (1) and (2) appropriately as well as supplement them with additional boundary conditions.

Consider first the case in which the normal phase behaves hydrodynamically, that is, when interparticle collisions are frequent enough for local thermodynamic equilibrium to be ensured everywhere during the oscillation. The chemical potential and pressure remain well-defined quantities and therefore Eqs. (1) and (2), with $\bar{\mu}$ and $\bar{P}$ replaced by $\bar{\mu}+\delta \mu$ and $\bar{P}+\delta P$, respectively, remain applicable.

In the system under study, particles may pass over from the $\mathrm{N}$ to the SF phase and vice versa [5]. This interconversion separately conserves the mass of each species. Denoting the position of the interface by $\zeta=\bar{\zeta}+\delta \zeta$, where $\delta \zeta$ is the departure of the interface from the equilibrium position, the appropriate boundary conditions (one for each of $i=\uparrow, \downarrow$ ) are, at each point on the interface,

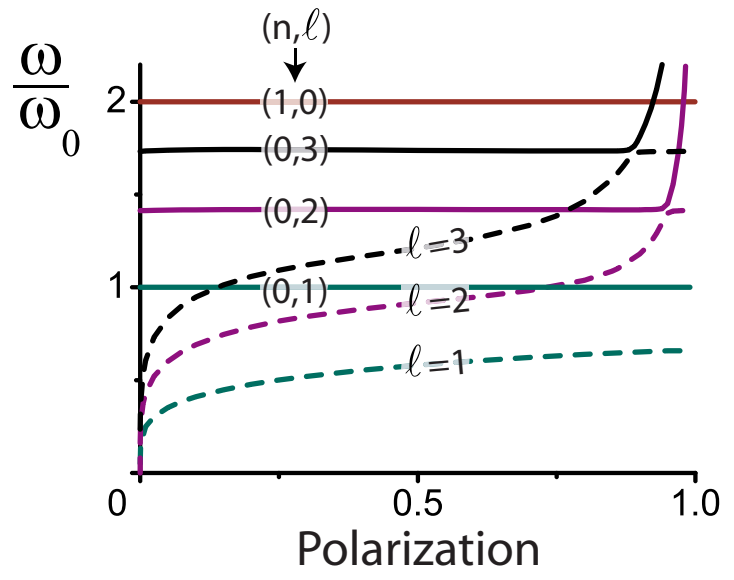

FIG. 2. (Color online) Collective mode frequencies against polarization for a trap with a hydrodynamic normal shell and with 7 $\times 10^{6}$ particles. The dashed and full lines denote the out-of-phase and in-phase collective modes, respectively. The frequencies of the in-phase modes are rather insensitive to the polarization, except for polarizations after the avoided crossing. At zero polarization, the IP modes reduce to the excitations of the single-component systems, labeled by the numbers $n$ and $\ell$.

$$
\mathbf{e}_{\zeta} \cdot\left(2 \mathbf{v}_{i} \rho_{i}-\mathbf{v}_{S F} \rho_{S F}\right)_{\zeta}=\left(2 \rho_{i}-\rho_{S F}\right)_{\zeta} \partial_{t} \delta \zeta,
$$

in which $\boldsymbol{e}_{\zeta}$ is the unit vector perpendicular to the interface (and directed toward the $\mathrm{N}$ side) and $\mathbf{v}$ are the velocities. These boundary conditions are, essentially, a restatement of the continuity equation for the case of two coexisting phases of different densities with a movable, permeable interface separating them.

Finally, the bulk dynamics for both the SF and the $\mathrm{N}$ are governed by the Euler and continuity equations. Applying standard techniques [13], we linearize these and obtain the equation for the deviation of the chemical potential from its equilibrium value, $\delta \mu$. Taking the temporal and angular parts of $\delta \mu_{S F}, \delta \mu_{\uparrow}, \delta \mu_{\downarrow}$, and $\delta \zeta$ to be $e^{i \omega t}$ and $\Phi$, respectively, we obtain $\delta \mu_{S F} \propto r^{\ell} F\left(\alpha^{+}, \alpha^{-}, \alpha^{0},\left(r / R_{S F}\right)^{2}\right) \quad$ and $\delta \mu_{i}$ $\propto r^{\ell} F\left(\alpha^{+}, \alpha^{-}, 3 / 2,1-\left(r / R_{i}\right)^{2}\right)$ with $i=\uparrow, \downarrow$, and $F$ the hypergeometric function. Here $\alpha^{0}=\ell+3 / 2,2 \alpha^{ \pm}=\ell+2 \pm\left[\ell^{2}+\ell\right.$ $\left.+4+3 \omega^{2} / \omega_{0}^{2}\right]^{1 / 2}$, and $\Phi=\mathrm{Y}_{l, m}$, with $\ell$ a positive integer or zero. For all three phases, we have also defined $R_{j}^{2}$ $\equiv 2 \mu_{j}^{0} / m \omega_{0}^{2}$, where $j=\uparrow, \downarrow$, or SF. The linearized versions of Eqs. (1)-(3), together with the linearized bulk solutions, now suffice to describe the dynamical behavior of the system in the case where the normal side is hydrodynamic [7].

In Fig. 2 we show the dependence of the mode frequencies on the polarization. We distinguish between the two kinds of excitations already mentioned: the in-phase (IP) modes, analogous to the excitations present in singlecomponent systems (full lines), and the out-of-phase (OOP) modes, unique to two-component systems (dashed lines). Note that similar excitations appear in trapped boson-boson systems [14]. At zero polarization, the IP mode frequencies reduce to the single-component frequencies $\omega^{2} / \omega_{0}^{2}=\ell$ $+2 n[(2 / 3)(n+\ell+1 / 2)+1][15]$, which are in almost exact agreement with the experiments [11]. For all polarizations, the frequency of the sloshing (or Kohn) mode remains ex- 
actly $\omega=\omega_{0}$; this excitation corresponds to a rigid cloud motion and is therefore not affected by the bulk equation of state. The frequencies of the IP modes with $n=0$ and $\ell>1$, on the other hand, vary slightly with polarization; this is a consequence of the nonzero interface tension. We note here that recent experiments at MIT reveal the existence of a large partially polarized shell resulting from the interactions in the $\mathrm{N}$ phase. The incorporation of such interactions is beyond the scope of this work. Nevertheless, our main result, the existence of the OOP modes, remains valid there since such modes are generic to the two-component system [14].

At temperatures well below the Fermi energy, a normal Fermi gas is expected to behave collisionlessly, especially in the case when this phase is fully spin polarized. We therefore turn our attention to a collisionless normal gas, that is, one that is described by a Boltzmann-Vlasov equation, as used, for example, to study a (spatially mixed) Bose-Fermi system in Ref. [16]. The issue of collective excitations in normal fermion gases has been addressed using several methods which include the hydrodynamic approximation, the method of averaging, the scaling ansatz method, the sum-rule approach, and the random-phase approximation [17].

The fully polarized normal gas is now described by a distribution function $f(\mathbf{r}, \mathbf{v}, t)$, evolving according to the Boltzmann-Vlasov equation

$$
\partial_{t} f+\boldsymbol{v} \cdot \nabla_{r} f-\omega_{0}^{2} \mathbf{r} \cdot \nabla_{u} f=0,
$$

rather than the hydrodynamic equations. We shall again consider small deviations from the equilibrium function, taking $f$ to deviate from the equilibrium value by a nonisotropic deformation of the Fermi surface [18]: $f(\mathbf{r}, \mathbf{p}, t)=f_{0}(\mathbf{r}, \mathbf{p}, t)$ $+\delta\left(|\mathbf{v}|-v_{F}\right) \nu(\mathbf{r}, \mathbf{p}, t)$, with $f_{0}$ the Fermi function and $v_{F}$ the (position-dependent) Fermi velocity. The interesting question then arises of which boundary conditions apply at an interface between a hydrodynamic and collisionless gas [11]. First, notice that, since we no longer have local thermodynamic equilibrium, there is no analog of Eq. (1) for the deviations from the equilibrium configuration. On the other hand, the Laplace condition, Eq. (2) remains valid with the following modifications: (a) since the normal gas is fully polarized, only quantities pertaining to the majority species appear in it; (b) the role of the pressure fluctuation of the majority species, $\delta P_{\uparrow}$ in the previous section, is now played by the radial component of the momentum flux tensor $\delta \Pi_{r r}^{\uparrow}$ $=m^{4} \int d^{3} \boldsymbol{v} v_{r}^{2}\left(f-f_{0}\right) /(2 \pi \hbar)^{3}$.

Next, from Eq. (3), we see that the radial component of the SF velocity must be equal to the radial velocity of the interface, that is, $\mathbf{e}_{\zeta} \cdot \mathbf{v}_{S F}=\partial_{t} \delta \zeta$, so that there is no flux through the interface. There are therefore only two possibilities for a particle incoming on the interface from the $N$ side: Andreev reflection or specular reflection [5]. Here, Andreev reflection is suppressed by the full spin polarization in the $\mathrm{N}$ phase, leaving specular reflection as the sole reflection mechanism. According to Bekharevich and Khalatnikov [21], specular reflection off a moving interface results in the boundary condition $\nu(-\chi)=\nu(\chi)-2 i \omega \chi \delta \zeta$, where $\chi=\cos \phi$ and $\phi$ the angle between $\mathbf{r}$ and $\mathbf{v}$. At $r=\bar{\zeta}$ the rotationally

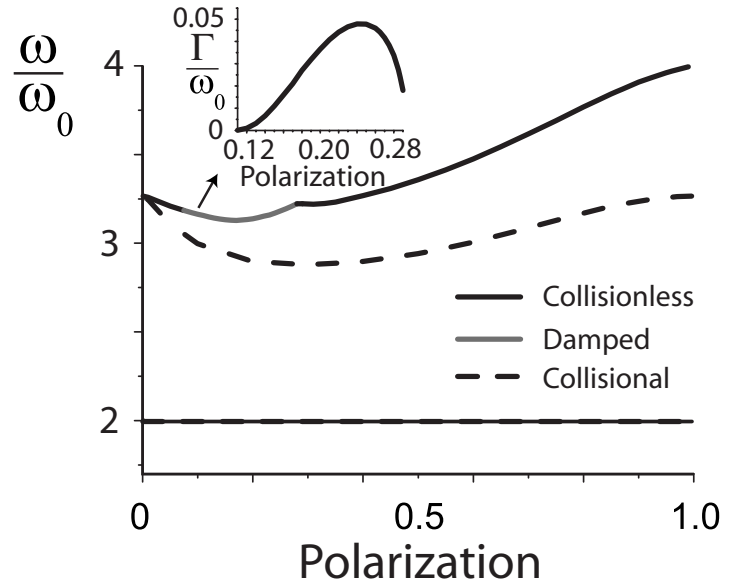

FIG. 3. Monopole frequencies against polarization for a trap with $7 \times 10^{6}$ particles. The lowest mode frequencies are exactly at $2 \omega_{0}$, both for the cases of a collisionless (full line) and hydrodynamic (dashed line) normal shell. The second monopole frequencies deviate substantially between these case. The gray full line indicates the region of damping for the collisionless case and the damping rates are shown in the inset.

symmetric $(\ell=0)$ solution satisfying Eq. (4) and this boundary equation is

$$
\nu(\bar{\zeta}, \chi, t)=\chi \omega \delta \zeta[\cot (\omega \tau / 2)+i],
$$

where $\tau$ is the time for a classical particle of velocity $\mathbf{v}$ to travel from the interface and back [19].

In Fig. 3, we present the monopole frequencies and compare the results of the collisionless (full lines) with those of the hydrodynamic approach for the normal shell. For both approaches, the lowest monopole frequency is $2 \omega_{0}$ for all polarizations; this is in agreement with an exact result derived by Castin [8] and differs from the results of Ref. [20]. As expected for the collisionless case, the second monopole frequency is $\sqrt{32 / 3} \omega_{0}$ at zero polarization and $4 \omega_{0}$ at full polarization. We find, however, that the collisionless mode is damped for polarizations between 0.09 and 0.28 (gray full line). The origin of this damping is a resonance which occurs when $\tau=2 \pi / \omega$; that is, when there exist particles for which the time to travel from the interface and back equals the period of the collective motion. Mathematically, it corresponds to the pole in $\nu$ [see Eq. (5)]. The damping frequency is obtained by writing $\omega \rightarrow \omega-i \Gamma$ with $\omega$ and $\Gamma$ real and positive, and analytically continuing the pressure tensor $\Pi_{r r}$ from negative to positive values of $\Gamma[7,22]$. As is shown in the inset of Fig. 3, the maximal damping for this number of particles is $\Gamma=0.05 \omega_{0}$, which is weak, but experimentally detectable. We stress that the origin of this damping is purely geometrical, and its presence and strength will strongly depend on the trapping geometry.

We have established the boundary conditions at the interface between the SF core and the surrounding N shell for a trapped imbalanced fermion gas in an isotropic trap at ultralow temperatures. Using these conditions we have obtained the frequencies of collective modes, as a function of 
the polarization, when the normal gas behaves hydrodynamically. We have also calculated the frequencies of the monopole mode for a collisionless normal gas.

For the hydrodynamic case, we find collective modes analogous to those in a single-component system, but with shifted frequencies. In addition, there exists a new class of collective modes; some of these have energies lower than the trap frequency. These modes correspond to out-of-phase motion of the SF core and the surrounding $\mathrm{N}$ shell.

For the case of a collisionless normal gas, we find that the lowest monopole mode remains at $\omega=2 \omega_{0}$, in agreement with an exact result due to Castin. We have also calculated the next lowest mode, finding that it reduces to the appropri- ate limits for vanishing and complete polarization of the trap and, interestingly, that it is damped for some polarizations. This damping is a geometric effect. It would be interesting to study crossover behavior of the OOP modes from the hydrodynamic to the collisionless normal gas.

We acknowledge partial support by Project No. FWO G.0115.06; B.V.S. and A.L. are supported by Project No. GOA/2004/02. We thank Joseph Indekeu for a careful reading of the manuscript and Theja De Silva for bringing Ref. [8] to our attention. We also thank Bidzina Shergelashvili for useful discussions.
[1] M. W. Zwierlein, A. Schirotzek, C. H. Schunck, and W. Ketterle, Science 311, 492 (2006); C. H. Schunck, Y. Shin, A. Schirotzek, M. W. Zwierlein, and W. Ketterle, ibid. 316, 867 (2007); M. W. Zwierlein, C. H. Schunck, A. Schirotzek, and W. Ketterle, Nature (London) 442, 54 (2006).

[2] G. B. Partridge, W. Li, R. I. Kamar, Y. A. Liao, and R. G. Hulet, Science 311, 503 (2006); G. B. Partridge, W. Li, Y. A. Liao, R. G. Hulet, M. Haque, and H. T. C. Stoof, Phys. Rev. Lett. 97, 190407 (2006).

[3] T. N. De Silva and E. J. Mueller, Phys. Rev. Lett. 97, 070402 (2006).

[4] M. Haque and H. T. C. Stoof, Phys. Rev. Lett. 98, 260406 (2007).

[5] B. Van Schaeybroeck and A. Lazarides, Phys. Rev. Lett. 98, 170402 (2007).

[6] H. Zhai and D.-H. Lee, e-print arXiv:cond-mat/07090388.

[7] A. Lazarides and B. Van Schaeybroeck (unpublished).

[8] Y. Castin, C. R. Phys. 5, 407 (2004).

[9] J. Carlson and S. Reddy, Phys. Rev. Lett. 95, 060401 (2005); T. D. Cohen, ibid. 95, 120403 (2005); F. Chevy, ibid. 96, 130401 (2006).

[10] The interface tension accounts for the most important corrections to the local density approximation [4].

[11] S. Giorgini, L. P. Pitaevskii, and S. Stringari, e-print arXiv:cond-mat/07063360, Rev. Mod. Phys. (to be published).

[12] Unitarity allows us to write $\sigma=\eta m \mu_{S F}^{2} / \hbar^{2}$, with $\eta$ a dimensionless quantity depending on the local interface curvature. Haque and Stoof find $\eta=0.6$, independently of the polariza- tion, therefore independently also of the curvature [4].

[13] C. J. Pethick and H. Smith, Bose-Einstein Condensation in Dilute Gases (Cambridge Press, Cambridge, 2002).

[14] A. A. Svidzinsky and S. T. Chui, Phys. Rev. A 67, 053608 (2003); 68, 013612 (2003).

[15] A. Bulgac and G. F. Bertsch, Phys. Rev. Lett. 94, 070401 (2005); H. Heiselberg, Phys. Rev. Lett. 93, 040402 (2004).

[16] T. Maruyama, H. Yabu, and T. Suzuki, Phys. Rev. A 72, 013609 (2005); T. Maruyama and G. F. Bertsch, e-print arXiv:0706.1128.

[17] G. M. Bruun and C. W. Clark, Phys. Rev. Lett. 83, 5415 (1999); D. Guéry-Odelin, F. Zambelli, J. Dalibard, and S. Stringari, Phys. Rev. A 60, 4851 (1999); D. Guéry-Odelin, ibid. 66, 033613 (2002); M. Amoruso, I. Meccoli, A. Minguzzi, and M. P. Tosi, Eur. Phys. J. D 7, 441 (1999); L. Vichi and S. Stringari, Phys. Rev. A 60, 4734 (1999); G. M. Bruun, ibid. 63, 043408 (2001); P. Capuzzi and E. S. Hernandez, ibid. 63, 063606 (2001).

[18] E. M. Lifshitz and L. Pitaevskii, Statistical Mechanics, Part 2 (Pergamon Press, Oxford, 1980).

[19] We find $\tau=\arctan [2 \chi /(\widetilde{r} / \zeta-\zeta / \widetilde{r})] / \omega_{0}$ and $\tilde{r}^{2}=R_{\uparrow}^{2}-\zeta^{2}$.

[20] T. N. De Silva and E. J. Mueller, e-print arXiv:cond-mat/ 0607491.

[21] I. L. Bekarevich and I. M. Khalatnikov, Zh. Eksp. Teor. Fiz. 39, 1699 (1960) [Sov. Phys. JETP 12, 1187 (1961)].

[22] L. Landau, J. Phys. (USSR) 1, 25 (1946); P. M. Bellan, Fundamentals of Plasma Physics (Cambridge University Press, Cambridge, 2006). 